\title{
Quantum jumps between dressed states: a proposed cavity-QED test using feedback
}

\author{
J. E. Reiner \\ Centre for Quantum Dynamics, School of Science, Griffith University, Brisbane 4111, Australia. \\ Department of Physics and Astronomy, SUNY Stony Brook, Stony Brook NY 11794-3800, USA. \\ H. M. Wiseman \\ Centre for Quantum Dynamics, School of Science, Griffith University, Brisbane 4111, Australia. \\ H. Mabuchi \\ Norman Bridge Laboratory of Physics 12-33, California Institute of Technology, Pasadena, CA 91125, USA.
}

(October 29, 2002)

\begin{abstract}
A strongly driven cavity containing a single resonant strongly coupled atom exhibits a phase bistability. The phase of the field is strongly correlated with the phase of the atomic dipole. It has been shown previously that phase-sensitive monitoring of the field emitted by the cavity would induce conditional quantum jumps between orthogonal atomic dipole states ("dressed" states). Here we show that such monitoring can be used to fix the atom into a single dressed state. As soon as a state-changing quantum jump is inferred from the measurement of the field, the atomic state is flipped using a $\pi$-pulse. We study this feedback scheme analytically and numerically. We show that the occupation probability of the desired fixed state can be as high as $1-1 / 8 \eta C_{1}$, where $C_{1} \gg 1$ is the single-atom co-operativity and $\eta$ the detection efficiency (which does not have to be close to unity). The control of the atomic dynamics is manifest in the fluorescence spectrum. The widths of all three peaks are modified from the usual Mollow spectrum, and almost all of the area under one of the sidebands is transferred to the other sideband. This is as expected, as one of the dressed states is essentially unoccupied, and transitions out of it do not occur. In addition, the width of the central peak goes to zero. This indicates coherent scattering due to the non-zero mean atomic dipole created by the feedback.
\end{abstract}

\section{INTRODUCTION}

Bohr's quantum jumps between atomic states [1] were the first form of quantum dynamics to be postulated. Of course Bohr's old theory did not survive the quantum revolution of the $1920 \mathrm{~s}$. In its aftermath, quantum jumps were revived [2] with a new interpretation as state reduction caused by measurement. However, simple quantum jump models for atoms were never entirely forgotten. For example, the dressed state model [3] was used successfully to give an intuitive explanation of the Mollow triplet [4] in resonance fluorescence.

It was the electron shelving experiments of Itano and co-workers [5] which focussed attention on the conditional dynamics of individual atoms. Subsequent work on waiting time distributions $[6,7]$ led to a renewal of interest in quantum jump descriptions [8]. It was shown by Carmichael [9] that quantum jumps are implicit in standard photodetection theory. Around the same time, stochastic quantum jump equations were introduced as a tool for simulating the dynamics of a dissipative system with a large Hilbert space $[10,11]$, and their links with quantum measurement theory were also noted $[12,13]$. This measurement interpretation is generally known as quantum trajectory theory $[9,14]$. By adding filter cavities as part of the apparatus, even the quantum jumps in the dressed state model can be interpreted as approximations to measurement-induced jumps [15].

The measurement interpretation of quantum trajectories has proven invaluable for understanding and predicting quantum optical correlation functions, especially in certain cavity QED experiments $[16,17]$. However, correlation functions traditionally were, and always can be, calculated within a deterministic formalism $[18,19]$. Is there more direct evidence for the existence of interesting quantum states conditioned upon continuous monitoring? In Ref. [20] one of us suggested that "conditioning is realized by feedback". That is, the way to see a conditioned state is by using the measurement results on which it is conditioned in a feedback loop to change the system dynamics [21]. This has recently been realized by two of us and co-workers [22] in a weakly driven cavity QED system. In that experiment, changing the driving field a precise time after the detection of a photon from the cavity freezes the conditioned state until the driving is returned to its initial value, when it resumes its evolution.

This paper proposes another way of realizing conditioning by feedback, this time in a strongly driven cavity QED system. We assume a single, strongly coupled $\left(C_{1}=g^{2} / \kappa \gamma \gg 1\right)$ atom resonant with the cavity. This system was shown $[23,24]$ to exhibit optical phase bistability, with the phase of the field strongly correlated with the phase of the atomic dipole. The case of many atoms was shown to exhibit optical phase multistability [25]. Previously, two of us [26] have shown quantitatively how phase-sensitive monitoring of the field emit- 
ted by the cavity would enable one to infer the atomic state. This was also noted (in a different context) in Ref. [27]. Such a measurement would cause "retroactive" [26] quantum jumps between orthogonal atomic dipole states ("dressed" states), as predicted by the dressed atom model [3]. As noted above, these jumps could be induced directly (not retroactively) by measuring the atomic fluorescence using filters. But in practice, the efficiency of such a measurement would be so low that the cavity QED scheme is much more likely to be realized experimentally.

In this paper we show that with feedback, one of these conditioned dressed states can be stabilized. The idea is simply to flip the atomic state using a $\pi$ pulse as soon as a state-changing quantum jump is inferred from the measurement of the field. We consider the simplest measurement and feedback scheme, and obtain quantitative predictions for how well the atomic state can be fixed in one dressed state. This turns out to be only weakly dependent upon $\eta$, unlike other feedback-stabilization schemes (see Ref. [28] and references therein). We also calculate the complete spectrum of the feedback-modified atomic fluorescence, which shows the enhancement of one sideband at the expense of the other. This is also as would be predicted from the dressed atom model [3]. This experiment would thus be a test for the conditional states predicted by this model, and, more importantly, a test of the conditional states predicted by the full quantum trajectory theory.

This paper is structured as follows. In Sec. II we introduce the cavity QED system, and various ways to describe it. This includes a semiclassical picture of the field and atomic dipoles, an approximate quantum description which reflects this, and a quantum description of the atom alone in the regime where the field can be slaved to the atom. In Sec. III we investigate the stochastic conditional dynamics in the latter two descriptions. Based on this understanding of the system, in Sec. IV we propose our feedback scheme. We show that for this scheme we do wish to work in the slaved-field regime, so that the system can be modelled by the state of the atom alone. Using a feedback-modified master equation for the atom we obtain quantitative predictions for the atomic state and the resonance fluorescence spectrum in Sec. V. We compare this with the results of a numerical simulation of the full system. We conclude with a discussion in Sec. VI.

\section{THE SYSTEM}

The cavity QED system we consider is defined by a single, two-level atom (TLA) strongly coupled to a single mode of a Fabry-Perot cavity. This system is well described by the Jaynes-Cummings Hamiltonian [18,19]

$$
H_{J C}=\hbar \omega_{a} \sigma_{z}+\hbar \omega_{c} b^{\dagger} b-i \hbar g\left(\sigma^{\dagger} b-b^{\dagger} \sigma\right),
$$

where $\sigma^{\dagger}, \sigma$, and $\sigma_{z}=\left[\sigma, \sigma^{\dagger}\right]$ are the Pauli spin operators for raising, lowering, and inversion of the atom, and $b^{\dagger}$ and $b$ are the standard raising and lowering operators for the internal cavity field mode with frequency $\omega_{c}$. The strength of the atom-field coupling is characterized by the dipole coupling constant, $g$, and is given by

$$
g=\sqrt{\frac{\mu^{2} \omega_{a}}{2 \hbar \epsilon_{0} V}},
$$

where $\mu$ is the transition dipole moment, $\omega_{a}$ is the atomic transition frequency, and $V$ is the cavity-mode volume.

The cavity QED system radiates energy through two channels. The first channel is along the cavity axis (i.e. through the cavity mirrors) and causes decay of the field amplitude at the rate of $\kappa$. The second channel is spontaneous emission from the two level atom into modes other than the cavity mode. This causes decay of the atomic dipole at the rate of $\gamma / 2$, where we assume the atomic radiative decay is essentially unmodified by the cavity. We use the single atom co-operativity $C_{1}=g^{2} / \kappa \gamma$ to describe the strength of the coherent atom-field coupling relative to the rate of decay of the system through these two channels. We consider the strong coupling regime $C_{1} \gg 1$ throughout this paper.

Figure 1 presents a simplified picture of the single atom cavity QED setup. We define the driving field as $\mathcal{E}$, meaning that in a frame rotating at the driving laser frequency it produces a Hamiltonian

$$
H_{d}=-i \hbar \mathcal{E}\left(b-b^{\dagger}\right)
$$

We assume that the driving laser frequency is on resonance with both the cavity and atomic transitions $\left(\omega_{l}=\right.$ $\left.\omega_{c}=\omega_{a}\right)$.

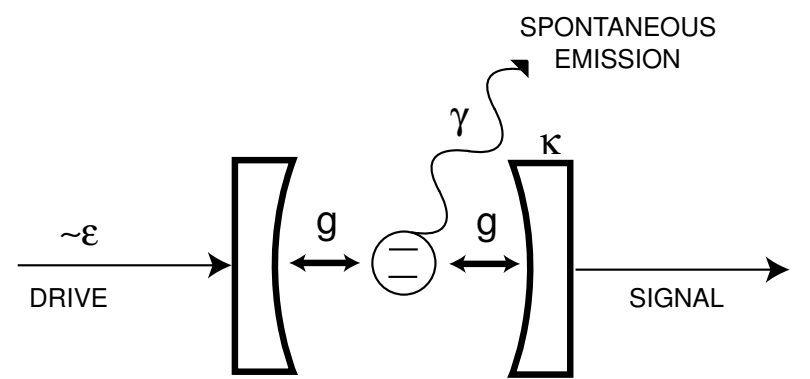

FIG. 1. An illustration of the single-atom cavity QED system.

\section{A. Master Equation}

The closed cavity QED system is well described by the Jaynes-Cummings Hamiltonian in Eq. (2.1). Performing measurements on any system requires opening the system to environmentally induced fluctuations. This leads to dissipation which modifies the Jaynes-Cummings 
dynamics. If the system interacts weakly and homogeneously with many modes of the environment such that the Born-Markov approximation is valid then these environmental degrees of freedom may be traced out to leave a modified system evolution $[19,29]$. This evolution is described by a quantum master equation of the Lindblad [30] type,

$$
\dot{\rho}=-i[H, \rho]+\sum_{\mu=1}^{M} \mathcal{D}\left[c_{\mu}\right] \rho \equiv \mathcal{L} \rho,
$$

where $M$ is the number of environmental channels available for the system to decay through. Given arbitrary operators $A$ and $B$, the superoperator $\mathcal{D}$ is given by

$$
\mathcal{D}[A] B \equiv A B A^{\dagger}-\left(A^{\dagger} A B+B A^{\dagger} A\right) / 2 .
$$

We incorporate $\gamma$ and $\kappa$ into the master equation with the following substitutions,

$$
\begin{aligned}
& c_{1}=\sqrt{\gamma} \sigma, \\
& c_{2}=\sqrt{2 \kappa} b .
\end{aligned}
$$

Combining Eqs. (2.1), (2.3) and substituting Eqs. (2.6) and (2.7) into Eq. (2.4) leads to the following expression for the quantum master equation for an open cavity QED system,

$$
\dot{\rho}=\left[g\left(b^{\dagger} \sigma-\sigma^{\dagger} b\right)-i \mathcal{E} y, \rho\right]+2 \kappa \mathcal{D}[b] \rho+\gamma \mathcal{D}[\sigma] \rho,
$$

where $y \equiv-i b+i b^{\dagger}$ is the phase quadrature of the cavity field and therefore $x \equiv b+b^{\dagger}$ is the amplitude quadrature.

\section{B. Semiclassical Fixed Points}

We begin our analysis of Eq. (2.8) by reviewing the semiclassical results in the large field regime obtained by Alsing and Carmichael [23].

Constructing the equations of motion for the semiclassical variables, $\alpha=\langle b\rangle, s=\langle\sigma\rangle$, and $w=\left\langle\sigma_{z}\right\rangle$, we find

$$
\begin{aligned}
\dot{\alpha} & =-\kappa \alpha+\mathcal{E}+g s, \\
\dot{s} & =g w \alpha-\frac{1}{2} \gamma s, \\
\dot{w} & =-2 g\left(\alpha^{*} s+s^{*} \alpha\right)-\gamma(w+1) .
\end{aligned}
$$

We assume that $\gamma$ is so small as to be negligible. Then the atomic steady state will be pure: $\left\langle\sigma_{x}^{2}\right\rangle+\left\langle\sigma_{y}^{2}\right\rangle+\left\langle\sigma_{z}^{2}\right\rangle=1$. This condition can be recast in terms of the variables $w$ and $s$ as

$$
w^{2}+4|s|^{2}=1
$$

where we used the fact that $\sigma_{y}=-i\left(\sigma^{\dagger}-\sigma\right)$ and $\sigma_{x}=$ $\left(\sigma^{\dagger}+\sigma\right)$.

There exist two sets of fixed points for Eqs. (2.9-2.11) in the $\gamma=0$ case. One corresponds to $\alpha_{\mathrm{fix}}=0$ (for
$2 \mathcal{E}<g$ ) and the other to $w_{\text {fix }}=0$ (for $2 \mathcal{E}>g$ ). Since we are interested in the limit of high driving, we consider the latter case which leads to the following set of fixed points.

$$
\begin{aligned}
\alpha_{\text {fix }}^{ \pm} & =\frac{\mathcal{E}+g s_{\text {fix }}^{ \pm}}{\kappa} \\
s_{\text {fix }}^{ \pm} & =-\frac{g}{4 \mathcal{E}} \mp i \sqrt{\frac{1}{4}-\left(\frac{g}{4 \mathcal{E}}\right)^{2}}, \\
w_{\text {fix }} & =0 .
\end{aligned}
$$

In the strong driving limit, $\mathcal{E} \gg g$, these expressions simplify to

$$
\begin{aligned}
\alpha_{\mathrm{fix}}^{ \pm} & =\frac{\mathcal{E}}{\kappa} \mp \frac{i g}{2 \kappa} \equiv \bar{\alpha} \mp i(g / 2 \kappa), \\
s_{\mathrm{fix}}^{ \pm} & =\mp \frac{i}{2}
\end{aligned}
$$

These semiclassical fixed points correspond to the following set of quantum states

$$
\left|\psi_{\text {fix }}^{ \pm}\right\rangle=\left|\alpha_{\text {fix }}^{ \pm}\right\rangle 2^{-1 / 2}[|g\rangle \mp i|e\rangle] \equiv\left|\alpha_{\text {fix }}^{ \pm}\right\rangle| \pm\rangle,
$$

where $\left|\alpha_{\text {fix }}^{ \pm}\right\rangle$is a coherent state [18].

We simplify further analysis by separating the mean coherent component of the cavity field from its fluctuations. Rewriting Eq. (2.8) explicitly in terms of $a=b-\bar{\alpha}$,

$$
\begin{aligned}
\dot{\rho}= & {\left[g\left(a^{\dagger} \sigma-\sigma^{\dagger} a\right)-i(\Omega / 2) \sigma_{y}, \rho\right]+\gamma \mathcal{D}[\sigma] \rho } \\
& +2 \kappa \mathcal{D}[a] \rho,
\end{aligned}
$$

where $\Omega=2 g \mathcal{E} / \kappa$ is the Rabi frequency.

Figure 2 shows the $Q$ distribution [18] for the cavity field in steady state. Notice the bimodal structure which corresponds to the two fixed points in Eq. (2.13).

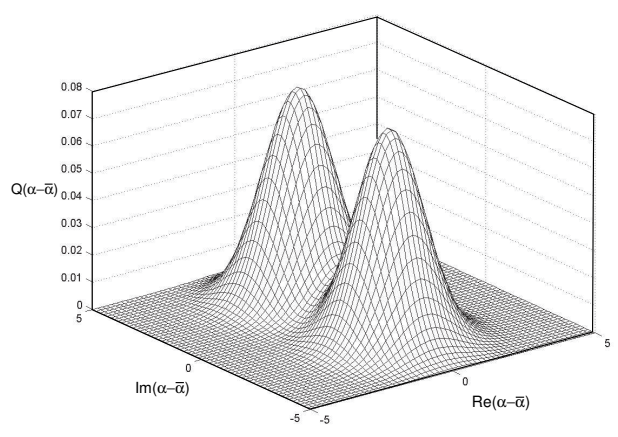

FIG. 2. The $\mathrm{Q}(\alpha-\bar{\alpha})$ distribution calculated numerically from Eq. (2.19) in steady state. The bimodal structure of this distribution illustrates the two fixed points for the cavity field. These fixed points are separated along the phase quadrature by $g / \kappa$. Parameters used for this calculation are $(g, \kappa, \Omega) / \gamma=(60,20,1200)$. These parameters were chosen so as to show the clear separation of the fixed points. 


\section{Secular Approximation for Rabi frequency $\Omega$}

In this section we show that the semiclassical fixed points of the preceding section are central to the full quantum dynamics in the limit where $\Omega$ is much greater than all other relevant rates. Apart from its last part, where we derive an explicit expression for the steady state $\rho$, this section is taken from Ref. [26].

Consider the dynamics in the interaction picture with respect to the Rabi Hamiltonian $H_{0}=\Omega \sigma_{y} / 2$. This changes Eq. (2.19) into

$$
\dot{\bar{\rho}}=\left[g\left(a^{\dagger} \bar{\sigma}-\bar{\sigma}^{\dagger} a\right), \bar{\rho}\right]+2 \kappa \mathcal{D}[a] \bar{\rho}+\gamma \mathcal{D}[\bar{\sigma}] \bar{\rho}
$$

where the bar indicates the operator is in the interaction picture. The transformed atomic lowering operator is

$$
\bar{\sigma}(t)=-\frac{i}{2}\left(\mu e^{-i \Omega t}+\mu_{z}-\mu^{\dagger} e^{i \Omega t}\right)
$$

where $\mu=|+\rangle\langle-|, \mu_{z}=\left[\mu^{\dagger}, \mu\right]=\sigma_{y}$. Notice that the cavity field is invariant under this transformation.

Substituting Eq. (2.21) into the first term of Eq. (2.20) and assuming that $\Omega \gg g$ allows the use of the rotating wave approximation (RWA). This lets us ignore the rapidly oscillating terms in the Jaynes-Cummings Hamiltonian. This leads to the following simplification of Eq. (2.20)

$$
\dot{\bar{\rho}}=-i g / 2\left[x \mu_{z}, \bar{\rho}\right]+2 \kappa \mathcal{D}[a] \bar{\rho}+\gamma \mathcal{D}[\bar{\sigma}] \bar{\rho} .
$$

This simplified master equation will be used in Sec. II D in our discussion of the adiabatic elimination.

Continuing on, we now substitute Eq. (2.21) into the third term of Eq. (2.22). We apply the RWA to arrive at

$$
\begin{aligned}
\dot{\bar{\rho}}= & -i g / 2\left[x \mu_{z}, \bar{\rho}\right]+2 \kappa \mathcal{D}[a] \bar{\rho} \\
& +(\gamma / 4)\left(\mathcal{D}[\mu]+\mathcal{D}\left[\mu_{z}\right]+\mathcal{D}\left[\mu^{\dagger}\right]\right) \bar{\rho} .
\end{aligned}
$$

Figure 3 illustrates the transitions described by the last terms in Eq. (2.23). The $\mathcal{D}[\mu]$ and $\mathcal{D}\left[\mu^{\dagger}\right]$ correspond to the atom flipping from the $|-\rangle$ to $|+\rangle$ states, and the reverse, respectively. These transitions correspond to the upper and lower sidebands of the atom's fluorescence spectrum respectively. The $\mathcal{D}\left[\mu_{z}\right]$ terms corresponds to transitions between the same atomic dressed states. If the atom is in one dressed state then the rate of statechanging jumps and non-state-changing jumps are both $\gamma / 4$. This gives a total rate of spontaneous emission of $\gamma / 2$, as expected for a strongly driven atom (which is half-excited).

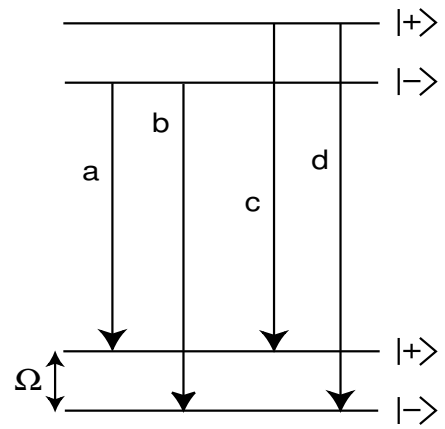

FIG. 3. Energy level diagram for the dressed state of the atom. Transitions $a$ and $d$ correspond to the $\mathcal{D}[\mu]$ and $\mathcal{D}\left[\mu^{\dagger}\right]$ switching terms respectively. Transitions $b$ and $c$ correspond to the $\mathcal{D}\left[\mu_{z}\right]$ term and are on resonance with the driving field.

Now consider the following ansatz [26] for the density operator $\bar{\rho}$,

$$
\bar{\rho}=\sum_{s= \pm}|s\rangle\left\langle s\left|\otimes \int_{-g / \kappa}^{g / \kappa} d y P_{s}(y)\right| i y / 2\right\rangle\langle i y / 2|,
$$

where $|i y / 2\rangle$ is a coherent state for the operator $a$. This ansatz assumes the cavity field can be described with a Glauber-Sudarshan coherent field distribution $[18,19]$ on a line connecting the two semiclassical fixed points of different phases. It also assumes that the atomic state is diagonal in the dressed-state basis, and that its state may be correlated with the phase of the field. Substituting Eq. (2.24) into Eq. (2.23) shows that these assumptions are correct, and leads to the following dynamic equations for $P_{ \pm}$, the field $P$-functions associated with the the $| \pm\rangle$ atomic states,

$\dot{P}_{ \pm}(y)=\frac{\partial}{\partial y}( \pm g+\kappa y) P_{ \pm}(y)+(\gamma / 4)\left[-P_{ \pm}(y)+P_{\mp}(y)\right]$.

The probability for the atom to occupy the state $|s\rangle$ is given by $p_{s}=\int d y P_{s}(y)=\operatorname{Tr}[\rho|s\rangle\langle s|]$. In steady state, we find that

$$
\begin{aligned}
& P_{+}{ }^{\mathrm{ss}}(y)=C(g-\kappa y)^{\gamma / 2 \kappa}(g+\kappa y)^{\gamma / 2 \kappa-1}, \\
& P_{-}{ }^{\mathrm{ss}}(y)=C(g-\kappa y)^{\gamma / 2 \kappa-1}(g+\kappa y)^{\gamma / 2 \kappa},
\end{aligned}
$$

where $C$ is a normalization constant. It is worth noting that in the $\gamma \rightarrow 0$ limit we recover the semiclassical fixed points as $P_{ \pm}^{\mathrm{ss}}(y) \rightarrow \delta(y \mp g / \kappa)$.

Figure $4 \mathrm{a}$ and b show plots of Eqs. (2.26) and (2.27) for two regimes. These illustrate the distribution of the field states for this system. Notice that the field is only defined in the region $-g / \kappa \leq y \leq g / \kappa$. We see that in the limit with $\gamma<2 \kappa$ that the field distributions are centered around the fixed points. In the other limit with $\gamma>2 \kappa$ we see that the atomic states are not as well centered on the fixed points of the field. We will study stochastic dynamics in Sec. III to further illustrate the dynamics that lead to these distributions. 

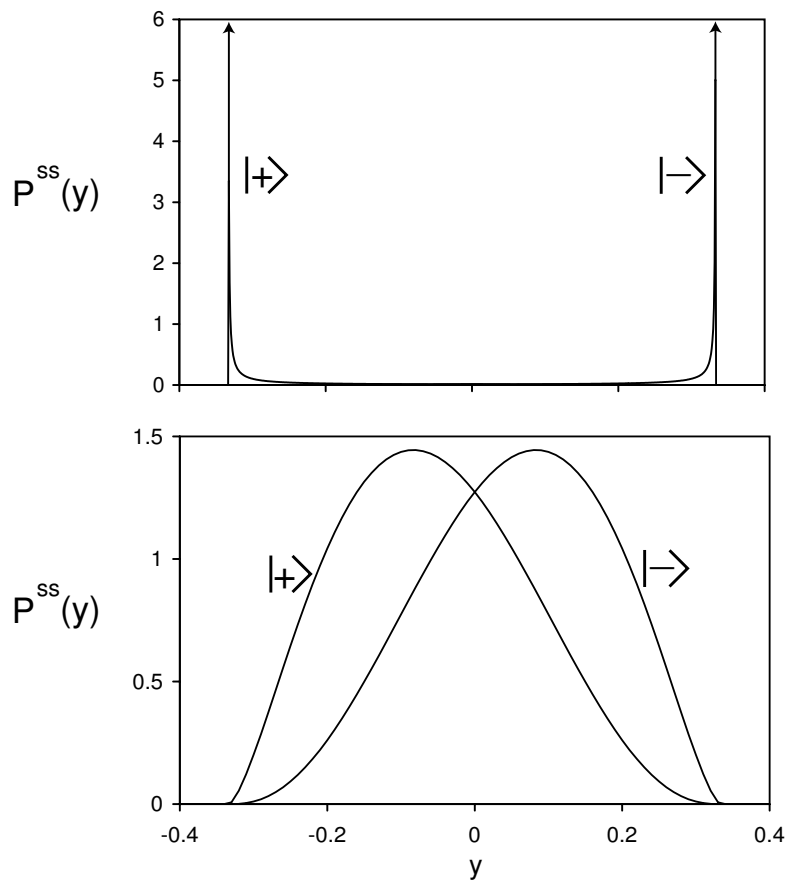

FIG. 4. Steady state distributions of the cavity field for two different values of $\gamma$. The top graph corresponds to the semiclassical fixed point limit with $\gamma / 2 \kappa=0.067$. The lower graph shows the mixing of the field states when $\gamma / 2 \kappa=2.5$. $g / \kappa=0.33$ for both plots.

\section{Adiabatic Approximation}

As we will show in Sec. IV, it turns out that for the feedback protocol we propose it is necessary to have $\kappa$ much larger than $g$ and $\gamma$. In this limit the cavity field dynamics will be slaved to the atomic dipole. This allows for the adiabatic elimination of the cavity field as in Ref. [39]. We begin by expanding the density operator in the field state basis to include the zero and one photon excitations.

$$
\bar{\rho}=\bar{\rho}^{0}|0\rangle\langle 0|+\left(\bar{\rho}^{1}|0\rangle\langle 1|+\text { H.c. }\right)+\bar{\rho}^{2}|1\rangle\langle 1| .
$$

Substituting Eq. (2.28) into Eq. (2.22) leads to the following set of equations:

$$
\begin{aligned}
& \dot{\bar{\rho}}^{0}=-i g\left(\mu_{z} \bar{\rho}^{1 \dagger}-\bar{\rho}^{1} \mu_{z}\right) / 2+\gamma \mathcal{D}[\bar{\sigma}] \bar{\rho}^{0}+2 \kappa \bar{\rho}^{2}, \\
& \dot{\bar{\rho}}^{1}=-i g\left(\mu_{z} \bar{\rho}^{2}-\bar{\rho}^{0} \mu_{z}\right) / 2+\gamma \mathcal{D}[\bar{\sigma}] \bar{\rho}^{1}-\kappa \bar{\rho}^{1}, \\
& \dot{\bar{\rho}}^{2}=-i g / 2\left(\mu_{z} \bar{\rho}^{1}-\bar{\rho}^{\dagger \dagger} \mu_{z}\right)+\gamma \mathcal{D}[\bar{\sigma}] \bar{\rho}^{2}-2 \kappa \bar{\rho}^{2} .
\end{aligned}
$$

The atomic density operator is the full density operator traced over the cavity field, $\bar{\rho}_{a}=\operatorname{Tr}_{\mathrm{c}}(\bar{\rho})=\bar{\rho}^{0}+\bar{\rho}^{2}$. From Eq. (2.29) and Eq. (2.31) we find that $\dot{\bar{\rho}}_{a}$ is given by

$$
\dot{\bar{\rho}}_{a}=-i g / 2\left[\mu_{z}, \bar{\rho}^{1}+\bar{\rho}^{1 \dagger}\right]+\gamma \mathcal{D}[\bar{\sigma}] \bar{\rho}_{a} .
$$

Equation (2.30) is dominated by the linear term in $\kappa$. This term quickly damps the cavity mode and we ignore initial transients so that $\dot{\bar{\rho}}^{1}=0$. To leading order this leads to

$$
\bar{\rho}^{1}=i \frac{g}{2 \kappa} \bar{\rho}^{0} \mu_{z} .
$$

In effect, we have slaved the cavity field state, determined to leading order by the off-diagonal $\bar{\rho}^{1}$, to the atomic state, determined to leading order by $\bar{\rho}^{0}$.

Substituting Eq. (2.33) into Eq. (2.32) gives the following expression (to leading order) for the master equation of the atom alone.

$$
\dot{\bar{\rho}}_{a}=\frac{g^{2}}{2 \kappa} \mathcal{D}\left[\mu_{z}\right] \bar{\rho}_{a}+\gamma \mathcal{D}[\bar{\sigma}] \bar{\rho}_{a} .
$$

Transforming Eq. (2.34) out of the interaction picture recovers the original driving term.

$$
\dot{\rho}_{a}=\gamma \mathcal{D}[\sigma] \rho_{a}+\frac{g^{2}}{2 \kappa} \mathcal{D}\left[\sigma_{y}\right] \rho_{a}-i \Omega\left[\sigma_{y}, \rho_{a}\right] \equiv \mathcal{L}_{a} \rho_{a} .
$$

We thus see that the bad cavity limit allows for the adiabatic elimination of the cavity field, yielding a simplified master equation for the two-level atom alone. The first term describes spontaneous emission events that occur at the slowest rate $\gamma$. The field contributes the second and third terms. The second term corresponds to a quantum non-demolition (QND) measurement on the state of the atom. This "measurement" can be viewed as the lossy cavity introducing a noisy driving term in combination with the strong driving given by the third term. This is shown in more detail in appendix A.

\section{STOCHASTIC DYNAMICS}

\section{A. Quantum Trajectories}

A brief review of the theory of quantum trajectories is provided in appendix B, to which we refer readers unfamiliar with this field.

We begin unraveling Eq. (2.20) with direct cavity detections by introducing the following cavity "jump" operator,

$$
\mathcal{J} \bar{\rho}=2 \kappa a \bar{\rho} a^{\dagger} .
$$

Tracing over the cavity we arrive at an expression which describes the effect that Eq. (3.1) has on the atom.

$$
\operatorname{Tr}_{c}(\mathcal{J} \bar{\rho})=2 \kappa \bar{\rho}^{2} .
$$

In the bad cavity limit one can slave the populated ondiagonal cavity field element with the vacuum element by setting $\dot{\bar{\rho}}^{2}=0$. Then, to leading order, one finds the following expression for $\bar{\rho}^{2}$,

$$
\bar{\rho}^{2}=\frac{g^{2}}{4 \kappa^{2}} \mu_{z} \bar{\rho}_{a} \mu_{z} .
$$


Substituting Eq. (3.3) into Eq. (3.2) we find

$$
\operatorname{Tr}_{c}(\mathcal{J} \bar{\rho})=\frac{g^{2}}{2 \kappa} \mu_{z} \bar{\rho}_{a} \mu_{z}=\mathcal{J}_{a} \bar{\rho}_{a}
$$

which is equivalent to the "jumps" associated with the noisy QND measurement term from Eq. (2.34).

We continue by constructing the $(\mathcal{L}-\mathcal{J}) \bar{\rho}$ operator from Eq. (2.22)

$$
(\mathcal{L}-\mathcal{J}) \bar{\rho}=-i g / 2\left[x \mu_{z}, \bar{\rho}\right]-\kappa\left(a^{\dagger} a \bar{\rho}-\bar{\rho} a^{\dagger} a\right) .
$$

Substituting Eq. (2.28) into Eq. (3.5) we find that the non-jump evolution is described by

$$
\operatorname{Tr}_{c}[(\mathcal{L}-\mathcal{J}) \bar{\rho}]=-\frac{g^{2}}{2 \kappa} \bar{\rho}_{a}=\left(\mathcal{L}_{a}-\mathcal{J}_{a}\right) \bar{\rho}_{a}
$$

Equation (3.6) together with Eq. (3.4) demonstrate the equivalence of unraveling the full density operator with cavity detections and unraveling the atomic density operator with the $\mu_{z} \bar{\rho} \mu_{z}$ detections. Therefore, under the adiabatic approximation, monitoring the state of the cavity is equivalent to monitoring the state of the atom.

The above measurements are insensitive to the phase of the cavity field. For feedback we wish to distinguish different phases of the cavity field, and hence different atomic states. This requires interfering the light emitted from the cavity with a suitable local oscillator. An obvious possibility, considered in Ref. [26] is to use a large local oscillator to do homodyne detection. Atomic jumps could be detected by looking for positive or negative transitions in the filtered photocurrent, although the optimal signal extraction algorithm is much more complicated [26] andwould require processing the current using digital electronics, as in Ref. [31]. Analysis of the resulting non-Markovian feedback would be quite difficult. In this paper we consider a much simpler sort of feedback, which is Markovian and which is based upon detecting the cavity light after a weak local oscillator has been added.

Figure 5 illustrates how one could use a local oscillator to offset the large output cavity field. The intuitive idea is to set the phase and amplitude of the local oscillator so as to cancel the output of the cavity when the field is in the semiclassical fixed state $\left|\alpha^{+}\right\rangle$corresponding to the $|+\rangle$ dressed state of the atom. Detecting a single photon from the cavity plus local oscillator field indicates that the field has left that fixed state, so implying that the atom has undergone a jump from the desired dressed state $|+\rangle$ to the other $|-\rangle$.

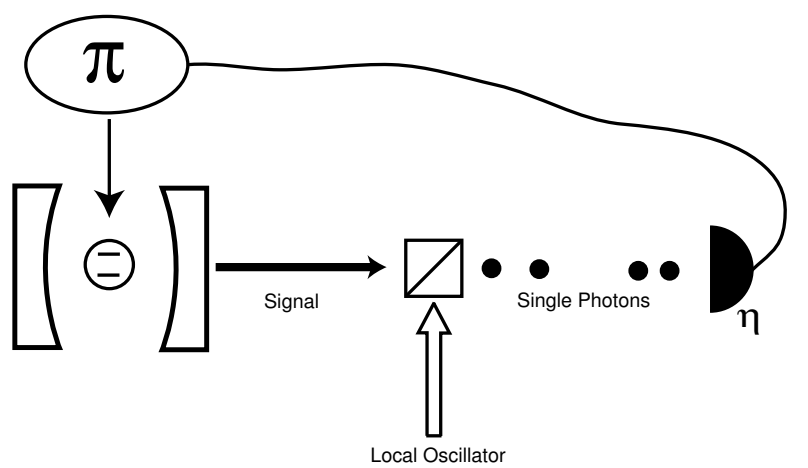

FIG. 5. The proposed direct detection scheme with a local oscillator to offset the large coherent field. The photodetector efficiency is given by $\eta$.

We have already added the real part of the local oscillator when we changed from the full field operator $b$ to the fluctuation field operator $a$. All that remains is to further offset the cavity field by an appropriate amount. If we wish to stabilize the atom in the $|+\rangle$ state, the extra field is equal to $\beta=i g / 2 \kappa$. The conditional dynamics in this case can be understood with the help of the identity

$$
\mathcal{D}[a] \rho=\mathcal{D}[a+\beta]-\frac{1}{2}\left[\beta^{*} a-\beta a^{\dagger}, \rho\right] .
$$

With the local oscillator in place, cavity jumps will now correspond to the following operator $[9,39]$

$$
\mathcal{J} \bar{\rho}=2 \kappa\left(a+\frac{i g}{2 \kappa}\right) \bar{\rho}\left(a^{\dagger}-\frac{i g}{2 \kappa}\right) .
$$

By tracing Eq. (3.8) over the cavity field and following a procedure similar to the one used to arrive at Eq. (3.4) we find that unraveling the master equation with Eq. (3.8) is equivalent to unraveling with the following "jump" operator

$$
\operatorname{Tr}_{\mathrm{c}}(\mathcal{J} \bar{\rho})=\frac{g^{2}}{2 \kappa}\left(\mu_{z}-1\right) \bar{\rho}_{a}\left(\mu_{z}-1\right) .
$$

For the atomic density operator, it turns out that

$$
\mathcal{D}\left[\mu_{z}\right] \rho_{a}=\mathcal{D}\left[\mu_{z}-1\right] \rho_{a} .
$$

Thus the rewritten master equation (transformed back out of the interaction picture) is simply

$$
\dot{\rho}_{a}=\gamma \mathcal{D}[\sigma] \rho_{a}+\frac{g^{2}}{2 \kappa} \mathcal{D}\left[\sigma_{y}-1\right] \rho_{a}-\frac{i \Omega}{2}\left[\sigma_{y}, \rho_{a}\right] .
$$

The new jump operator, which corresponds to detecting a photon as shown in Fig. (5), is $\sigma_{y}-1$. Note that this takes $|+\rangle$ to $|-\rangle$ as desired. When this happens, the detected photon can be used to trigger a $\pi$-pulse to take the atom back to state $|+\rangle$. This will be described in detail in Sec. IV. 


\section{B. Field Dynamics under the Secular Approximation}

The above analysis assumed $\kappa$ large enough to adiabatically eliminate the cavity field. We stated in Sec. II D that this was required for the particular feedback protocol in this paper. To justify this it is necessary to give up that assumption, and examine the stochastic dynamics of atom and field. This is tractable if we make the secular approximation of Sec. II C.

Imagine that we are constantly monitoring the cavity output along with all of the spontaneous emissions from the atom, and resolving the three peaks of the Mollow triplet. Then the total state will be a pure state, and from Eq. (2.24) the field will be in a coherent state $|i y / 2\rangle$ and the atom in one of the dressed states.

The final term in Eq. (2.23) shows the atom will jump between the $|+\rangle$ and $|-\rangle$ states at a rate of $\gamma / 4$. Assume that the atom is initially in the $|+\rangle$ dressed state and the field is $y=-g / \kappa$. From Eq. (2.23) we find the state of the cavity field following a jump into the $|-\rangle$ dressed state is given by

$$
\dot{y}=-(g+\kappa y),
$$

which implies that $y$ decays exponentially at rate $\kappa$ towards the other fixed point, $g / \kappa$. Each atomic state flip is followed by the cavity field reversing its direction of motion. Figure 6a demonstrates such a trajectory with $\gamma<2 \kappa$. The field spends most of the time near one or the other fixed point. If the rate of atomic flips is increased such that $\gamma>2 \kappa$, then we find a trajectory like the one shown in Fig. 6b. The field spends most of its time in between the two fixed points. These two figures provide the dynamic evolution which, upon ensemble averaging, leads to the steady state distributions shown in Fig. 4.
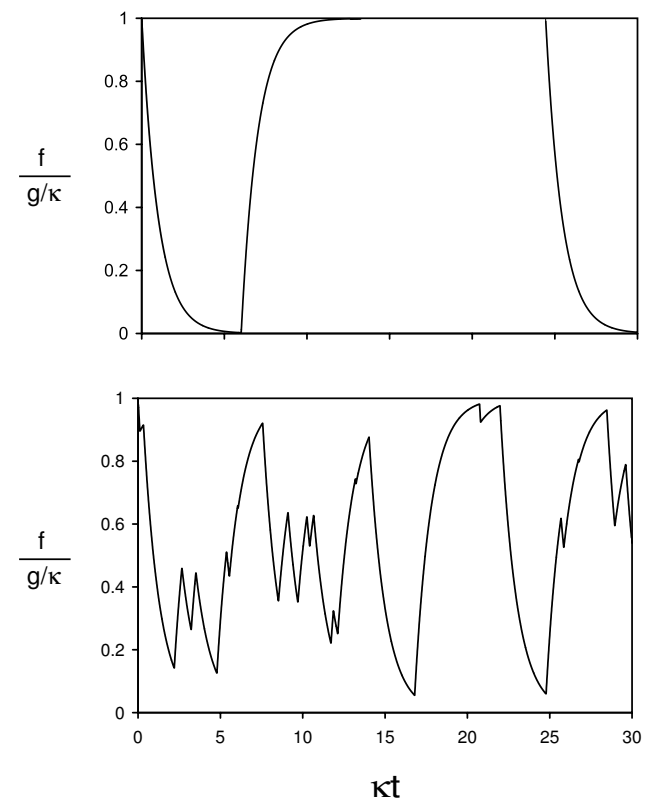

FIG. 6. Stochastic evolution of the cavity field following spontaneous emission events. The top figure corresponds to $\gamma / 2 \kappa /=2.5$ while the bottom figure corresponds to $\gamma / 2 \kappa=0.067$ which are the same values used to calculate the distributions in fig. 4. The parameter $f=y / 2+g / 2 \kappa$ is the field displacement from the $|+\rangle$ fixed point.

\section{FEEDBACK}

\section{A. Field Dynamics with Feedback}

We begin our feedback analysis in the secular approximation, to examine the cavity field dynamics. There are several time scales involved in our problem and in this subsection we establish what the relative sizes for these should be in order to give us the most effective feedback results.

Let the atom be in the $|+\rangle$ dressed state and the field in the corresponding fixed point coherent state $f=0$, where $f=y / 2+g / 2 \kappa$. With the weak local oscillator as described above, this means the detector sees a vacuum output for the cavity field. The feedback protocol involves switching the state of the atom only when we detect a photon from the cavity.

The following field dynamics illustrate a typical feedback event. Say the atomic state flips from $|+\rangle$ to $|-\rangle$ at $t=0$ by a spontaneous emission. Then the field $f$ will subsequently grow as

$$
f(t)=(g / \kappa)\left(1-e^{-\kappa t}\right) \text { for } 0 \leq t \leq t_{\mathrm{g}} .
$$

Here $t_{\mathrm{g}}$ is the time at which we first detect a cavity photon emission. Since (in this picture where the atomic state change is treated as a known event), the field is always in a coherent state, this detection has no effect on the conditional system state. However, with feedback, the detection triggers a $\pi$ pulse which switches the state of the atom. This "flip" will cause the field to reverse and head back towards the "vacuum" as

$$
f(t)=(g / \kappa)\left(1-e^{-\kappa t_{\mathrm{g}}}\right) e^{-\kappa\left(t-t_{\mathrm{g}}\right)} \text { for } t>t_{\mathrm{g}} .
$$

For long times, the system is restored to the desired state of $|+\rangle$ and $f=0$.

We consider two possible events that will disrupt the effectiveness of the feedback protocol. The first is a "bad" detection, following the "good" detection at time $t_{\mathrm{g}}$, as shown in Fig. 7. It is a bad detection because, with our simple feedback protocol (to switch the atomic phase whenever a cavity photon is detected), this detection will switch the atom back into the wrong dressed state $|-\rangle$. We wish to minimize the probability for this event.

The probability for a bad detection can be written as

$$
P_{\mathrm{b}}=\int_{0}^{\infty} d t_{\mathrm{g}} \int_{t_{\mathrm{g}}}^{\infty} d t_{\mathrm{b}} p_{\mathrm{b}}\left(t_{\mathrm{b}} \mid t_{\mathrm{g}}\right) p_{\mathrm{g}}\left(t_{\mathrm{g}}\right)
$$


Here $p_{\mathrm{b}}\left(t_{\mathrm{b}} \mid t_{\mathrm{g}}\right)$ is the conditional probability density for detecting a "bad" photon at time $t_{\mathrm{b}}$ following a "good" cavity detection at time $t_{\mathrm{g}}$. With a detection efficiency $\eta$, It is given by

$$
p_{\mathrm{b}}\left(t_{\mathrm{b}} \mid t_{\mathrm{g}}\right)=2 \kappa \eta\left[f\left(t_{\mathrm{b}}\right)\right]^{2},
$$

since the coherent field amplitude $f(t)$ depends upon $t_{\mathrm{g}}$ already in Eq. (4.2). Similarly, $p_{\mathrm{g}}\left(t_{\mathrm{g}}\right)$, the probability density for detecting the first photon at time $t_{\mathrm{g}}$, is given by

$$
p_{\mathrm{g}}\left(t_{\mathrm{g}}\right)=2 \kappa \eta\left[f\left(t_{\mathrm{g}}\right)\right]^{2} P\left(\text { no g before } t_{\mathrm{g}}\right) .
$$

Here $P$ (no g before $t_{\mathrm{g}}$ ), the probability for there to be no detections prior to $t_{\mathrm{g}}$, is equal to the solution of

$$
\dot{P}=-2 \kappa \eta[f(t)]^{2} P,
$$

with $P=1$ at $t=0$.

Using all of the above expressions we arrive at the following for the probability of a bad detection:

$$
P_{\mathrm{b}}=\frac{z^{2}}{2} \int_{0}^{\infty} d u[k(u)]^{2} \exp \left[-z \int_{0}^{u} d v k(v)\right],
$$

where we have defined the parameter $z=2 \eta g^{2} / \kappa^{2}$ and the function $k(u)=\left(1-e^{-u}\right)^{2}$. As stated, we wish to minimize this probability, which depends only upon $z$. It is not difficult to show that it is monotonically increasing with $z$, and is therefore minimized for small $z$. In this regime Eq. (4.7) reduces to

$$
P_{\mathrm{b}} \approx \frac{z}{2}=\frac{\eta g^{2}}{\kappa^{2}}
$$

Thus we require $g \ll \kappa$.

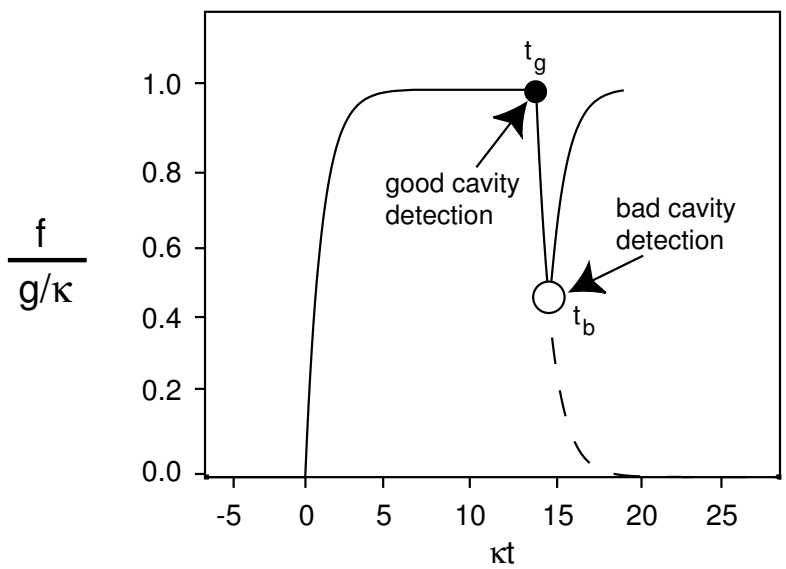

FIG. 7. Dynamics of the cavity field. A spontaneous emission flips the atomic state at $t=0$. This is detected from the "good" detection at time $t_{\mathrm{g}}$, and a feedback pulse applied. If a "bad" detection subsequently occurs at time $t_{\mathrm{b}}$ then an unwanted second feedback pulse is applied, which puts the atom in the wrong state and drives the cavity field in the wrong direction. The dashed line shows were the field should go without the bad detection at $t_{\mathrm{b}}$. As before the field is measured with the displaced operator $f=(y+g / \kappa) / 2$.
The second disrupting event occurs if the atomic state flips back to state $|+\rangle$ due to a spontaneous emission before its excursion into state $|-\rangle$ has been noticed through the detection of a cavity photon at time $t_{\mathrm{g}}$. At first glance this event seems to be helpful because it is forcing the atom back into the desired $|+\rangle$ dressed state. However, this ignores the fact that we wish to judge the success of the feedback by the elimination of the lower sideband in the Mollow triplet.

Figure 8 illustrates why this occurs if a feedback pulse forces the atom back into the proper state. If the atom flips back of its own accord, this corresponds to the $\mathcal{D}[\mu]$ term in Eq. (2.23) which means a photon emitted into the low energy sideband of the spectrum.

More generally, the occurrence of a second dipolechanging spontaneous emission before the first one has been noticed indicates that the measurement is failing to keep track of the state of the atom. We would thus expect this to have other, less obvious, adverse consequences for our ability to control the atomic state through feedback.

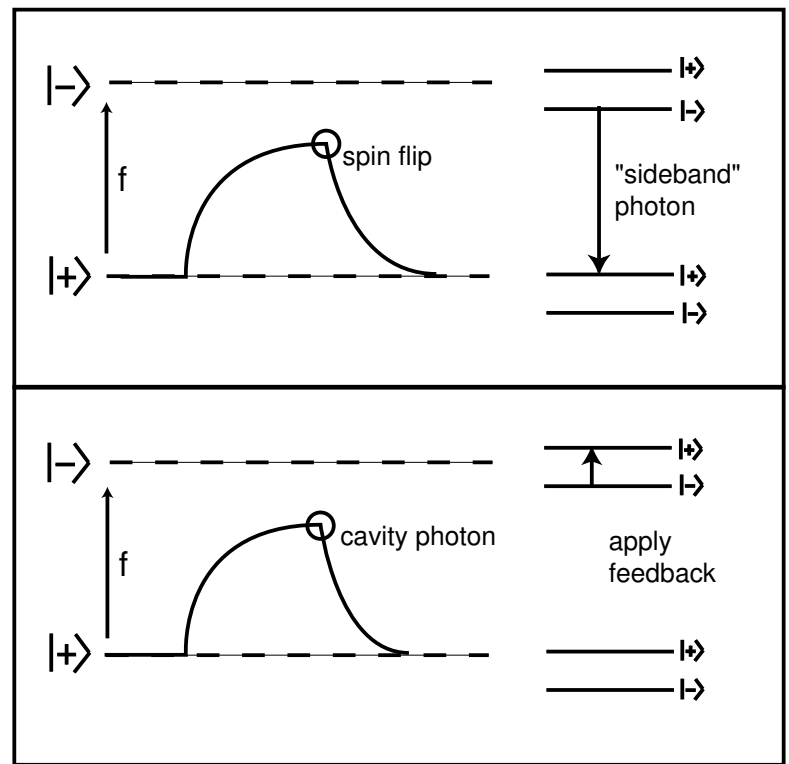

FIG. 8. Two possible events will keep the system in the $|+\rangle$ dressed state. The top event goes undetected by the feedback loop, but leads to an unwanted sideband fluorescence photon. The bottom event is detected and suppresses the sideband photon.

To suppress these events we first find, in a similar procedure as above, the full expression for the probability of the system undergoing a state flip before the cavity has emitted a photon at time $t_{\mathrm{g}}$. This is

$$
P_{\gamma}=1-\int_{0}^{\infty} d t_{\mathrm{g}} P\left(\text { no } \gamma \text { before } t_{\mathrm{g}}\right) p_{\mathrm{g}}\left(t_{\mathrm{g}}\right) .
$$

Since the rate of state-changing spontaneous emissions is $\gamma / 4$, the probability that there is no such event before time $t$ is simply given by 


$$
P(\text { no } \gamma \text { before } t)=e^{-\gamma t / 4} \text {. }
$$

Thus $P_{\gamma}$ evaluates to

$$
P_{\gamma}=1-z \int_{0}^{\infty} d u e^{-\gamma u / 2 \kappa} k(u) e^{-z \int_{0}^{u} d v k(v)} .
$$

This function is monotonically decreasing with the variable $z$. However, since we know from consideration of the first disrupting process that we require $z \ll 1$, we find an approximate analytic expression for $P_{\gamma}$ in the limit of small $z$,

$$
P \approx\left(1+\frac{2 \kappa z}{\gamma}\right)^{-1}
$$

For this to be small we evidently require $\gamma / \kappa \ll z$, or $\gamma \ll \eta g^{2} / \kappa$. This condition can be understood from the adiabatic equation (2.35) for the atomic state. This shows that the rate of the state-changing atomic jumps scales as $\gamma$, whereas QND measurement term (which is all that allows us to follow the state of the atom) has a strength scaling as $g^{2} / \kappa$. To follow the state of the atom well, we require the former to be small compared with the latter.

We finally combine all the inequalities that we have established so far for obtaining good feedback control of our two level system

$$
\Omega \gg \kappa \gg g \gg g^{2} / \kappa \gg \gamma .
$$

It is worth noting that Eq. (4.13) corresponds to the same inequalities that were assumed in order to justify the adiabatic elimination method in section II D. Also note that in order to minimize both $P_{\mathrm{b}}$ and $P_{\gamma}$, the optimal $g$ would scale as

$$
g \sim\left(\kappa^{3} \gamma / \eta^{2}\right)^{\frac{1}{4}}
$$

It should be noted that photo-detector dark counts will also be a source of error for the feedback scheme. We ignore them because their typical rate (less than $10^{2} \mathrm{~s}^{-1}$ ) is much less than the rate of photo-detections $\left(g^{2} / \kappa \sim 10^{7} \mathrm{~s}^{-1}\right)$. However, in practice there will be excess "dark" counts due to imperfect mode-matching between the cavity output beam and weak local oscillator. In addition, imperfect cavity locking (length stabilization) will lead to excess effective noise in the relative phase of the cavity output and local oscillator. Although this would have little deleterious effect on a homodyne scheme, it will contribute more dark counts to the adaptive photon counting scheme. This scheme would require interferometric stabilization of the entire optical setup, as in Ref. [32].

\section{B. Feedback Master Equation}

The general method for deriving a direct detection feedback master equation has been developed by one of us [21]. In the limit of Markovian feedback Eq. (2.8) is modified in the following way

$$
\dot{\rho}=-i[H, \rho]+\mathcal{D}\left[U c_{f}\right] \rho+\sum_{\mu=1}^{M-1} \mathcal{D}\left[c_{\mu}\right] \rho .
$$

Here $U$ is the unitary feedback operator which acts following a detection from channel $c_{f}$.

In this proposal the desired feedback operator $U$ flips the state of the atom. This could be accomplished with the application of a $\pi$ pulse along a mode which differs from the cavity mode. This can be represented by the following operator

$$
U=i(|+\rangle\langle-|-|-\rangle\langle+|)=\sigma_{x} .
$$

This approach is valid providing the feedback is applied instantaneously, as we will assume for the remainder of the paper. This approximation will be valid in the limit where the duration of the feedback pulse is shorter than $1 / \Omega$, as $\Omega$ is the fastest relevant atomic frequency. If this were not possible, then a more complicated pulse would have to be applied in order to have the desired effect in the interaction frame rotating at frequency $\Omega$.

The feedback is conditioned upon detections of the cavity field in interference with the local oscillator. We fully restate the master equation Eq. (2.19) using the identity (3.7) which displays the decay channel with the local oscillator added:

$$
\begin{aligned}
\dot{\rho} & =\left[g\left(a^{\dagger} \sigma-\sigma^{\dagger} a\right)-i \Omega \sigma_{y} / 2-i g x / 2, \rho\right] \\
& +\gamma \mathcal{D}[\sigma] \rho+2 \kappa \mathcal{D}[a+i g / 2 \kappa] \rho .
\end{aligned}
$$

We condition our feedback upon detections of the field, $c_{f}=\sqrt{2 \kappa}(a+i g / 2 \kappa)$. Upon substitution of Eq. (4.16) into Eq. (4.17) as in and Eq. (4.15), we arrive at the following expression for the feedback master equation

$$
\begin{aligned}
\dot{\rho} & =\left[g\left(a^{\dagger} \sigma-\sigma^{\dagger} a\right)-i \Omega \sigma_{y} / 2-i g x / 2, \rho\right] \\
& +2 \kappa \mathcal{D}\left[\sigma_{x}(a+i g / 2 \kappa)\right] \rho+\gamma \mathcal{D}[\sigma] \rho .
\end{aligned}
$$

One further improvement of our analysis is the inclusion of the detector efficiency, $\eta$. This requires the following modification [21]

$$
\begin{aligned}
\dot{\rho} & =\left[g\left(a^{\dagger} \sigma-\sigma^{\dagger} a\right)-i \Omega \sigma_{y} / 2-i g x / 2, \rho\right] \\
& +2 \kappa \eta \mathcal{D}\left[\sigma_{x}(a+i g / 2 \kappa)\right] \rho \\
& +2 \kappa(1-\eta) \mathcal{D}[(a+i g / 2 \kappa)] \rho+\gamma \mathcal{D}[\sigma] \rho .
\end{aligned}
$$

\section{Adiabatic Feedback Master Equation}

We begin our analysis of Eq. (4.19) with the assumptions in Eq. (4.13). This permits the use of the adiabatic elimination that was discussed for the non-feedback master equation in Section II D. Following the same method 
used there, we first transform into the interaction picture defined by $H_{I}=\Omega \sigma_{y} / 2$ and make the RWA as was done to arrive at Eq. (2.22)

$$
\begin{aligned}
\dot{\bar{\rho}} & =-i g / 2\left[x\left(\mu_{z}+1\right), \bar{\rho}\right]+2 \kappa \eta \mathcal{D}\left[\bar{\sigma}_{x}(a+i g / 2 \kappa)\right] \bar{\rho} \\
& +2 \kappa(1-\eta) \mathcal{D}[(a+i g / 2 \kappa)] \bar{\rho}+\gamma \mathcal{D}[\bar{\sigma}] \bar{\rho} .
\end{aligned}
$$

Adiabatically eliminating the field and then transforming out of the interaction picture leads to the following feedback master equation for the atom alone

$$
\begin{aligned}
\dot{\rho}_{a} & =-i \Omega / 2\left[\sigma_{y}, \rho_{a}\right]+\gamma \mathcal{D}[\sigma] \rho_{a}+(1-\eta) \frac{g^{2}}{2 \kappa} \mathcal{D}\left[\sigma_{y}\right] \rho_{a} \\
& +\frac{\eta g^{2}}{2 \kappa} \mathcal{D}\left[\sigma_{x}\left(\sigma_{y}-1\right)\right] \rho_{a} .
\end{aligned}
$$

We study both the fluorescence spectrum of the atom and the steady state population of the $|+\rangle$ state. Both of these are obtained from the dynamic equations for the quantities $\langle\sigma\rangle$ and $\left\langle\sigma_{z}\right\rangle$. These are found to be

$$
\begin{aligned}
\langle\dot{\sigma}\rangle= & -\left(\frac{\gamma}{2}+\frac{g^{2}}{2 \kappa}+\frac{\eta g^{2}}{\kappa}\right)\langle\sigma\rangle-\left(\frac{g^{2}}{2 \kappa}-\frac{\eta g^{2}}{\kappa}\right)\left\langle\sigma^{\dagger}\right\rangle \\
& +\Omega\left\langle\sigma_{z}\right\rangle / 2-\frac{i \eta g^{2}}{\kappa} \\
\left\langle\dot{\sigma}_{z}\right\rangle= & -\Omega\left(\left\langle\sigma^{\dagger}\right\rangle+\langle\sigma\rangle\right)-\left(\gamma+\frac{g^{2}}{2 \kappa}\right)\left\langle\sigma_{z}\right\rangle-\gamma
\end{aligned}
$$

From these we find the steady state values for $\left\langle\sigma_{x}\right\rangle,\left\langle\sigma_{y}\right\rangle$ and $\left\langle\sigma_{z}\right\rangle$ to be

$$
\begin{aligned}
\left\langle\sigma_{x}\right\rangle_{\mathrm{ss}} & =\frac{2 \Omega \kappa^{2} \gamma}{\gamma^{2} \kappa^{2}+2 \Omega^{2} \kappa^{2}+3 \gamma \kappa g^{2}+2 g^{4}}, \\
\left\langle\sigma_{y}\right\rangle_{\mathrm{ss}} & =\left(1+\frac{\gamma \kappa}{4 \eta g^{2}}\right)^{-1}, \\
\left\langle\sigma_{z}\right\rangle_{\mathrm{ss}} & =\frac{\kappa \gamma\left(\kappa \gamma+2 g^{2}\right)}{\kappa^{2} \gamma^{2}+2 \Omega^{2} \kappa^{2}+3 \kappa \gamma g^{2}+2 g^{4}} .
\end{aligned}
$$

Note that only $\sigma_{y}$ is changed from its no-feedback value (zero) which is obtained by letting $\eta \rightarrow 0$.

\section{RESULTS}

We present both analytical and numerical results for our feedback protocol. Numerical studies were carried out by solving Eq. (4.19) with the quantum optics toolbox software for MATLAB [33]. Analytical calculations of Eq. (4.22) and Eq. (4.23) were performed with algebraic manipulation software (MAPLE). We find that in the proper limits defined by Eq. (4.13) the two methods are in agreement. The results we present are for more typical cavity QED values [34].

We begin by solving for the steady state population of the $|+\rangle$ state. This is a direct measure of the effectiveness of our feedback protocol. The steady state population is related to the steady state expectation of $\sigma_{y}$ by

$$
P_{+}=\left(\frac{1+\left\langle\sigma_{y}\right\rangle_{\mathrm{ss}}}{2}\right) .
$$

Figure 9 shows a plot of the steady state population as a function of the detector efficiency for parameters consistent with the adiabatic approximation. Note that in this regime the feedback is not sensitively dependent on the detection efficiency.

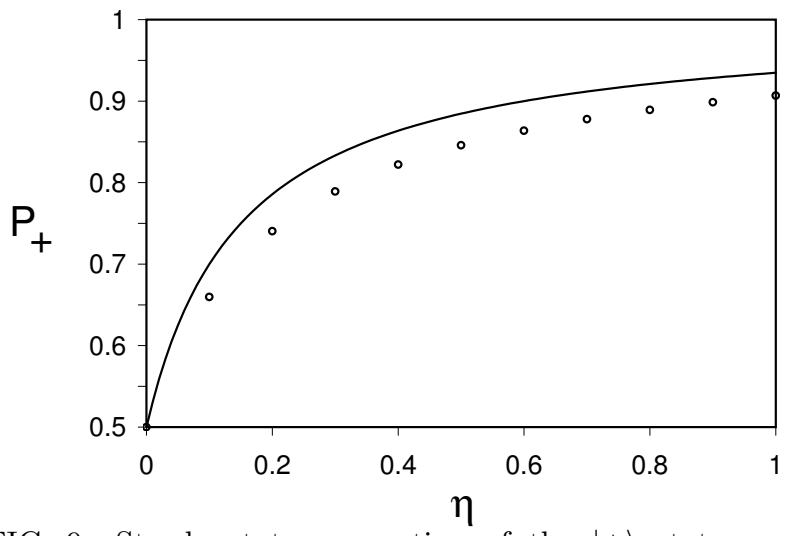

FIG. 9. Steady state occupation of the $|+\rangle$ state as a function of the detector efficiency. Numerical values are represented by dots and the approximate analytical expression is shown as a solid line throughout the rest of the paper. This plot was calculated with the following parameters: $(\Omega, \kappa, g) / \gamma=(50,15,5)$.

Figure 10 shows the population as a function of the ratio of the coupling, $g$, to the cavity decay rate $\kappa$. The agreement between the theory and numerical results is good in the limit of $g / \kappa \ll 1$, but fails at larger values of $g / \kappa$. This is as expected from Eq. (4.13), and also explains the discrepancy in Fig. 9. We also note that the effectiveness of this feedback begins to decrease as the values for $g$ are increased beyond $\kappa$. This is consistent with the arguments presented in section IV. A.

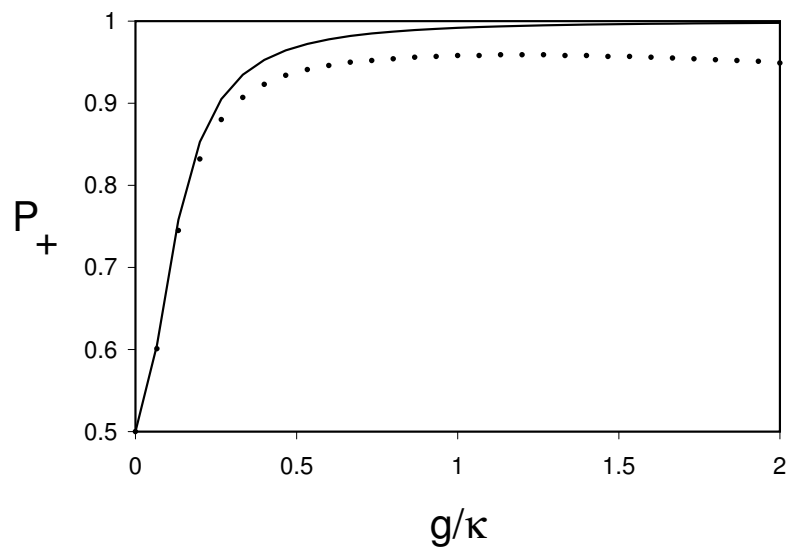

FIG. 10. Numerical calculation of the steady state occupation as a function of $g / \kappa$. The system parameters are the same as those used in Fig. 9 but with $\eta=1$. 
The fluorescence spectrum of the atom provides an experimentally accessible method for studying the effectiveness of this feedback. The spectrum of the atom is given explicitly by the Fourier transform of the two-time correlation function

$$
S(\omega)=\frac{\gamma}{2 \pi} \int_{-\infty}^{\infty} e^{-i \omega \tau}\left\langle\sigma^{\dagger}(\tau) \sigma(0)\right\rangle_{\mathrm{ss}} d \tau .
$$

An analytic expression for Eq. (5.2) follows by applying the quantum regression theorem to Eqs. (4.22) and (4.23). Using textbook methods [19] we arrive at an expression for the spectrum of the atom which is too lengthy to report. Instead we consider some of the more salient features of our results.

The spectrum, in the adiabatic regime, is described rather well by the sum of three Lorentzians and a $\delta$ function. Therefore it is the location, width, and area under these three peaks that is of most interest. Rewriting Eqs. (4.22-4.23) in matrix form,

$$
\dot{\mathbf{s}}=\mathbf{M s}
$$

with $\mathbf{s}=\left(\langle\sigma\rangle,\left\langle\sigma^{\dagger}\right\rangle,\left\langle\sigma_{\mathbf{z}}\right\rangle\right)^{\mathbf{T}}$, the position and widths of the three peaks are given by the eigenvalues of the $\mathbf{M}$ matrix,

$$
\begin{aligned}
& \lambda_{ \pm}=-3 \gamma / 4-g^{2} / \kappa \pm \sqrt{\gamma^{2} / 16-\Omega^{2}} \approx \Gamma_{1} \pm i \Omega \\
& \lambda_{0}=-\gamma / 2-2 g^{2} \eta / \kappa=\Gamma_{0} .
\end{aligned}
$$

where we have assumed that $\Omega \gg \gamma$, as required for the above equations to be valid.

Using these results, we write an approximate expression for the total spectrum of the atom in which the area under each peak is apparent.

$$
\begin{aligned}
S(\omega) & =A \delta(\omega)+\frac{B\left(\Gamma_{1} / \pi\right)}{\Gamma_{1}^{2}+(\omega+\Omega)^{2}} \\
& +\frac{C\left(\Gamma_{0} / \pi\right)}{\Gamma_{0}^{2}+(\omega)^{2}}+\frac{D\left(\Gamma_{1} / \pi\right)}{\Gamma_{1}^{2}+(\omega-\Omega)^{2}} .
\end{aligned}
$$

Here $A$ is the coherent $(\omega=0)$ component of the spectrum, coming from $\left\langle\sigma^{\dagger}\right\rangle_{\mathrm{ss}}\langle\sigma\rangle_{\mathrm{ss}}$. This is non-zero with the feedback precisely because the feedback stabilizes the atom in a state $|+\rangle$ with a definite dipole moment. To leading order this term evaluates to

$$
A=\gamma \frac{4 \eta^{2} C_{1}^{2}}{\left(1+4 \eta C_{1}\right)^{2}} \rightarrow \frac{\gamma}{4}
$$

Here the limit is for $\eta C_{1} \rightarrow \infty$, where $C_{1}=g^{2} / \kappa \gamma$ is the single atom co-operativity. The Lorentzian peaks at $\omega=-\Omega, \omega=0$ and $\omega=\Omega$ have areas $B, C$ and $D$ respectively. In the limit of large $\Omega$ they simplify to

$$
\begin{aligned}
& B=\frac{\gamma}{8} \frac{1}{1+4 \eta C_{1}} \rightarrow 0, \\
& C=\frac{\gamma}{4} \frac{1+8 \eta C_{1}}{\left(1+4 \eta C_{1}\right)^{2}} \rightarrow 0, \\
& D=\frac{\gamma}{8} \frac{1+8 \eta C_{1}}{1+4 \eta C_{1}} \rightarrow \frac{\gamma}{4} .
\end{aligned}
$$

Here the limits again are for $\eta C_{1} \rightarrow \infty$. In this limit we see that emission is divided equally between the $\omega=$ 0 coherent peak, and the $\omega=\Omega$ Lorentzian peak. By contrast, in the no feedback case $(\eta=0)$, one may quickly observe that the area under each of the sideband peaks is one-half that of the central peak. In all cases the sum of all peaks is equal to $\gamma / 2$.

Figures 11 and 12 show plots of the fluorescence spectrum of the atom derived analytically from the adiabatically eliminated master equation and numerically from the full master equation. Figure 11 has no feedback and the sidebands are equal in size. The scale of both plots was set to show the change in the relative sizes of the sidebands. By turning the feedback on in Fig. 11 we see that the low energy sideband is suppressed with the high energy sideband enhanced. Also notice the appearance of the $\delta$-function component on resonance. These features should be measurable experimentally.

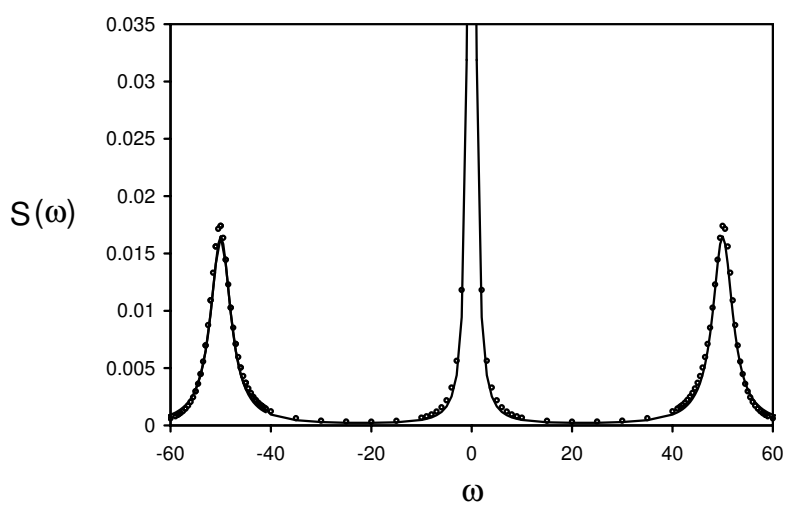

FIG. 11. Numerical (dots) and analytic (solid line) calculations for the fluorescence spectrum with no feedback. The parameters used are the same as in Fig. 9. Both axes are measured in units of $\gamma$.

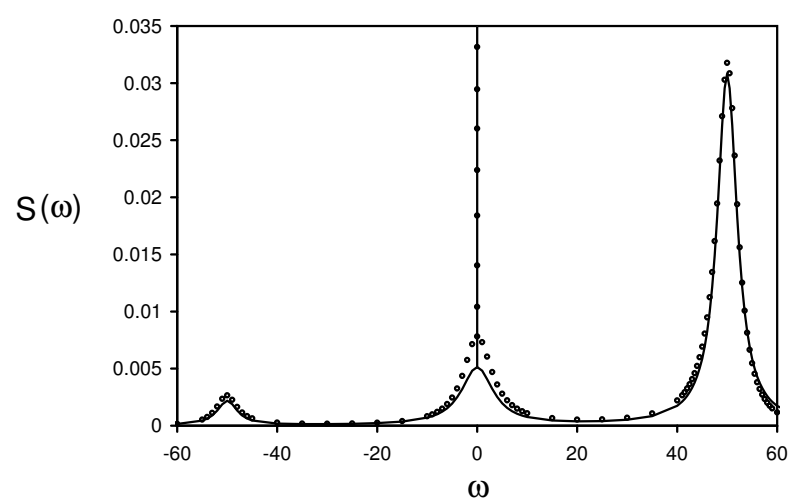

FIG. 12. Numerical (dots) and analytic (solid line) calculations for the fluorescence spectrum with feedback. Other details are as in Fig. 11. 


\section{DISCUSSION}

We have shown that feedback can be used to stabilize a strongly-driven atom in one dressed state, by reversing its polarization whenever its conditional state jumps into the other dressed state. The atomic state is conditioned upon phase-sensitive detection of the light emitted by a cavity mode which is strongly coupled to the atom. The cavity field acts as a QND measuring device for the atom. When the conditional state of the atom is forced to stay in one dressed state, one of the sidebands in the atomic fluorescence spectrum vanishes and the other doubles. These qualitative features are as would be predicted by the simple quantum jump model using dressed states [3]. However, to predict quantitatively the best regime, the effectiveness of the feedback, and the exact shape of the fluorescence spectrum, requires the rigorous quantum theory of feedback we have used here [21], based on quantum trajectories.

For our feedback scheme (which involves photon counting with a small local oscillator), the best regime is $\gamma \ll g^{2} / \kappa \ll g \ll \kappa \ll \Omega$. Homodyne detection, as considered in Ref. [26], could also be used as a basis for feedback although it would be more difficult to model and in some ways more difficult to implement experimentally. With homodyne-based feedback the considerations that led to the condition $g \ll \kappa$ do not obviously apply, and indeed in Ref. [26] the opposite condition held. However the condition $C_{1}=g^{2} / \kappa \gamma \gg 1$ would still be necessary, as the single-atom co-operativity determines how much the field is influenced by the atomic state.

In the regime $g \gg \kappa$, the field states correlated with the atomic polarization states have a phase difference much larger than the phase uncertainty of a coherent state. Hence they may be reliably distinguished and the cavity takes on the role of a meter, with distinct "pointer states" [35] correlated with orthogonal states of the microscopic system (atomic dipole). Indeed, coherent states with macroscopically different phases were one of the pointer states considered in the early work of Ref. [36]. However it is worth emphasizing that this macroscopic difference between states of the intracavity field is not necessary for feedback. In our regime, the two intracavity field states are barely distinct. Over timescales that are long compared to the cavity decay time, the light continuously leaking from the cavity reveals sufficient information (through continuous sampling) about the phase of the cavity field to enable the experimenter to discriminate between the two atomic states. Feedback stabilization of the atomic state is thus possible as long as the timescale for gaining this information is short compared to the average time between spontaneous emissions.

It is also interesting to compare our regime with that where $\kappa \gg \Omega$. In this regime it is possible to adiabatically eliminate the cavity without first making the secular ap- proximation in the frame rotating at Rabi frequency $\Omega$. Thus the cavity mode acts as a one-dimensional vacuum field for the atom [37], so detecting the light from the cavity is equivalent to detecting the atomic emission into the other vacuum modes. Since we assume $g^{2} / \kappa \gg \gamma$, emission into other modes can be ignored compared to emission through the cavity. In this regime, measuring the cavity emissions using exactly the same (small local oscillator) technique as we have proposed in this paper turns out to be practically identical to a measurement scheme proposed by one of us and Toombes [15] for detecting quantum jumps between dressed states. Thus the feedback scheme we have proposed here would work in principle irrespective of the ratio of $\kappa$ to $\Omega$. However, in the regime $\kappa \gg \Omega$ the purity of the conditioned state (and hence that of the feedback-stabilized state) would depend strongly upon the detection efficiency $\eta$. This is in contrast to the regime of this paper where the purity of the feedback-stabilized state depends only weakly upon $\eta$.

The fact that the effectiveness of the conditioning (and hence feedback) is not compromised by a detection efficiency less than unity is an attractive feature of the scheme we propose here. Paradoxically, other detection imperfections may even improve its effectiveness. Real detectors have finite dead-time following a detection, during which they cannot detect again. If this time were comparable to $g^{2} / \kappa$ then the probability of a "bad detection", as discussed in Sec. IV. A, would be much reduced. In fact, this could lift the $g \ll \kappa$ restriction derived in that section, and thereby make the realization of the experiment more flexible.

Feedback with a detector having a finite dead time could still be modeled relatively easily within the master equation formalism by using the theory of realistic detectors proposed by Warszawski and two of us [38]. The same theory could in principle be expanded to encompass the delay time and response function of the feedback loop. However, at some point the model would become so unwieldy that a quantum trajectory simulation would be the better option. A quantum trajectory simulation would also be the only practical way to simulate another experimental option, namely to use the feedback to flip the phase of the cavity field, rather than the phase of the atomic dipole. Since the dressed state is really defined by the relative phase of the atom and field, in principle this would have the same effect, and may be easier to achieve experimentally. The exploration of these experimental possibilities using quantum trajectory theory is a topic for future work. 


\section{APPENDIX A: EQUIVALENCE OF NOISY DRIVING AND ATOMIC QND MEASUREMENT}

We establish the equivalence of the $\mathcal{D}\left[\sigma_{y}\right]$ term in Eq. (2.35) and a noisy driving Hamiltonian. We begin by adding a stochastic noise term to Eq. (2.3)

$$
H_{d}(t)=\left(\Omega+\frac{g}{\sqrt{2 \kappa}} \xi(t)\right) \sigma_{y} .
$$

This stochastic term can be interpreted as the quantum noise in the amplitude of the cavity mode which is coupled to the atom. Eq. (2.35) is recovered by noting that $\rho_{a}^{c}+d \rho_{a}^{c}=e^{-i H d t} \rho_{a}^{c} e^{i H d t}$ and that the noisy term $\xi(t)$ in Eq. (6.1) obeys the usual Wiener increment statistics with $\langle\xi(t)\rangle=0$ and $\left\langle\xi(t)^{2}\right\rangle=1 / d t$.

$$
\begin{aligned}
\rho_{a}^{c}+d \rho_{a}^{c}= & -i\left[\Omega+\frac{g}{\sqrt{2 \kappa}} \xi(t), \rho_{a}^{c}\right] d t \\
& -\frac{g^{2}}{\kappa} \xi(t)^{2} d t^{2}\left(\sigma_{y}^{2} \rho_{a}^{c}+\rho_{a}^{c} \sigma_{y}^{2}-\sigma_{y} \rho_{a}^{c} \sigma_{y}\right),
\end{aligned}
$$

where $\rho_{a}^{c}$ is the conditioned density operator for the atom alone. Upon ensemble averaging over all possible trajectories we recover the last two terms in Eq. (2.35).

\section{APPENDIX B: BRIEF REVIEW OF QUANTUM TRAJECTORIES}

The simplest measurement-based unraveling of a master equation separates the evolution of the system into two parts [9]. The first is the jumps which correspond to detections at some detector outside the system. The second corresponds to the non-unitary but smooth evolution of the system between these jumps. A quantum trajectory is the evolution of the conditioned system state $\rho^{c}(t)$, consisting of alternating jumps and smooth evolution for various times. A weighted average over all possible conditioned evolutions leads to the unconditioned density operator, $\rho(t)$. We follow the presentation in Ref. [39] in providing a more quantitative discussion of these ideas.

We begin by stating the formal solution of Eq. (2.4)

$$
\rho(t)=e^{\mathcal{L} t} \rho(0) .
$$

The effect of a photodetection on the system state is described by

$$
\mathcal{J} \rho=c \rho c^{\dagger}
$$

We rewrite the master equation in terms of this jump superoperator, $\mathcal{L} \rho=\mathcal{J} \rho+(\mathcal{L}-\mathcal{J}) \rho$, and use a generalized Dyson expansion to separate out the two types of evolutions of the monitored system

$$
\rho(t)=\sum_{m=0}^{\infty} \int_{0}^{t} d t_{m} \int_{0}^{t_{m}} d t_{m-1} \ldots \int_{0}^{t_{2}} d t_{1} \tilde{\rho}^{c}(t) .
$$

with

$$
\tilde{\rho}^{c}(t)=\mathcal{S}\left(t-t_{m}\right) \mathcal{J S}\left(t_{m}-t_{m-1}\right) \ldots \mathcal{J} \mathcal{S}\left(t_{1}\right) \rho(0) .
$$

The unnormalized, conditioned density operator, $\tilde{\rho}^{c}(t)$, describes a particular series of jumps and smooth evolution according to $\mathcal{S}(t)=e^{(\mathcal{L}-\mathcal{J}) t}$. To normalize the conditioned density operator we simply divide by its trace, $\rho^{c}(t)=\tilde{\rho}^{c}(t) / \operatorname{Tr}\left[\tilde{\rho}^{c}(t)\right]$. This trace is also equal to the exclusive probability density for a particular series of photodetections, $p_{m}=\operatorname{Tr}\left[\tilde{\rho}^{c}(t)\right]$. Therefore, the unconditioned density operator becomes a weighted sum (or equivalently, ensemble average) over all trajectories:

$$
\begin{aligned}
\rho(t)=\sum_{m=0}^{\infty} \int_{0}^{t} d t_{m} & \int_{0}^{t_{m}} d t_{m-1} \ldots \\
& . \int_{0}^{t_{2}} d t_{1} p_{m}\left(t_{1}, \ldots, t_{m} ;[0, t]\right) \rho^{c}(t) .
\end{aligned}
$$

\section{ACKNOWLEDGMENTS}

HMW and JER wish to thank Paul Alsing and Luis Orozco respectively for discussions. This work was supported by the Australian Research Council and the NSF.

[1] N. Bohr, Phil. Mag. 26, 1 (1913).

[2] P. A. M. Dirac, The Principles of Quantum Mechanics (Oxford University Press, London, 1930).

[3] C. Cohen-Tannoudji and S. Reynaud, Phil. Trans. R. Soc. Lond. A 293, 223 (1979).

[4] B.R. Mollow, Phys. Rev. 188, 1969 (1969).

[5] J.C. Bergquist et al., Phys. Rev. Lett. 57, 1699 (1986).

[6] C. Cohen-Tannoudji and J. Dalibard, Europhys. Lett. 1, 441 (1986).

[7] P. Zoller, M. Marte and D.F. Walls, Phys. Rev. A 35, 198 (1987).

[8] H.J. Carmichael, S. Singh, R. Vyas, and P.R. Rice, Phys. Rev. A 39, 1200 (1989).

[9] H.J. Carmichael, An Open Systems Approach to Quantum Optics (Springer-Verlag, Berlin, 1993).

[10] R. Dum, P. Zoller and H. Ritsch, Phys. Rev. A 45, 4879 (1992).

[11] J. Dalibard, Y. Castin and K. Mølmer, Phys. Rev. Lett. 68, 580 (1992).

[12] C.W. Gardiner, A.S. Parkins, and P. Zoller, Phys. Rev. A 46, 4363 (1992).

[13] K. Mølmer, Y. Castin, and J. Dalibard, J. Opt. Soc. Am. B 10, 524 (1993).

[14] H. M. Wiseman and G. J. Milburn, Phys. Rev. A 47, 1652 (1993).

[15] H. M. Wiseman and G. E. Toombes, Phys. Rev. A 60, 2474 (1999). 
[16] H. J. Carmichael, H. Castro Beltran, G. T. Foster, and L. A. Orozco, Phys. Rev. Lett. 85, 1855 (2000).

[17] G. T. Foster, L. A. Orozco, H. M. Castro-Beltran, and H. J. Carmichael, Phys. Rev. Lett. 85, 3149 (2000).

[18] D. F. Walls and G. J. Milburn, Quantum Optics (Springer, Berlin, 1994).

[19] H.J. Carmichael, Statistical Methods in Quantum Optics vol. 1 (Springer-Verlag, Berlin, 1999).

[20] H. M. Wiseman, Phys. Rev. A 47, 5180 (1993).

[21] H.M. Wiseman, Phys. Rev. A 49, 2133 (1994); Errata ibid., 495159 (1994) and ibid. 50, 4428 (1994).

[22] W. P. Smith, J. E. Reiner, L. A. Orozco, S. Kuhr, and H. M. Wiseman, Phys. Rev. Lett. 89, 133601 (2002).

[23] P. Alsing and H.J. Carmichael, Quantum Opt. 3, 13 (1991)

[24] P. Alsing, Ph.D. thesis, University of Arizona, Tucson, Arizona, (1991).

[25] S.Y. Kilin and T.B. Krinitskaya, Phys. Rev. A 48, 3870 (1993).

[26] H. Mabuchi and H.M. Wiseman, Phys. Rev. Lett. 81, 4620 (1998).

[27] P. Kochan, H. J. Carmichael, P. R. Morrow, and M. G. Raizen, Phys. Rev. Lett. 75, 45 (1995).

[28] H.M. Wiseman, S. Mancini, and J. Wang, Phys. Rev. A 66, 013807 (2002).

[29] C. W. Gardiner and P. Zoller, Quantum Noise (Springer, Berlin, 2000).

[30] G. Lindblad, Commun. Math. Phys. 48, 199 (1976).

[31] M.A. Armen et al. Phys. Rev. Lett. 89, 133602 (2002).

[32] H. Mabuchi, J. Ye, and H.J. Kimble, App. Phys. B 68, 1095 (1999).

[33] S. Tan, Quantum Optics Toolbox for MATLAB, (1999).

[34] C.J. Hood et al. , Phys. Rev. Lett. 80, 4157 (1998).

[35] W. H. Zurek, Phys. Rev. D 24, 1516 (1981).

[36] D. F. Walls, M. J. Collett, and G. J. Milburn, Phys. Rev. D 32, 3208 (1985).

[37] Q.A. Turchette, R.J. Thompson, and H.J. Kimble, App. Phys. B 60, S1 (1995).

[38] P. Warszawski, H.M. Wiseman, and H. Mabuchi, Phys. Rev. A 65, 023802 (2002).

[39] H. M. Wiseman and G. J. Milburn, Phys. Rev. A 47, 642 (1993). 